\title{
ALTERAÇÕES NAS PROPRIEDADES QUÍMICAS DE UM SOLO ALUVIAL SALINO-SÓDICO DECORRENTES DA SUBSOLAGEM E DO USO DE CONDICIONADORES ${ }^{(1)}$
}

\author{
J. S. HOLANDA ${ }^{(2)}$, G. C. VITTI ${ }^{(3)}$, A. A. C. SALVIANO(4), \\ J. D. F. MEDEIROS(2) \& J . R. A. AMORIM(2)
}

\begin{abstract}
RESUMO
Solos afetados por sais são característicos de zonas áridas/semi-áridas. Este trabalho foi desenvolvido no vale do Açu (RN), no período abril/90 a janeiro/91, objetivando avaliar o efeito do preparo do solo (convencional e convencional + subsolagem) e da aplicação de condicionadores ( gesso, esterco de curral e pal ha de carnaúba) nas propriedades químicas de um solo aluvial salino sódico. A subsolagem proporcionou efeito eficaz na redução da porcentagem de sódio trocável (PST) e no abaixamento do pH do solo. Nos tratamentos com gesso, foram detectados os maiores valores de cálcio trocável, redução na PST para menos de $15 \%$ na camada de 0-0,15 m e aumento na salinidade do solo. 0 esterco de curral foi benéfico na redução da PST, principalmente quando associado à subsolagem e à gessagem. Existe um desbalanço natural nas relações $\mathrm{K}: \mathrm{Ca}: \mathrm{Mg}$ trocáveis no solo, que se acentua com a gessagem, necessitando de correção potássica para que se ati nja o equilíbrio. A proporção entre cátions trocáveis e solúveis no solo ficou entre 13 e 16:1, para cálcio e magnésio, e entre 0,3 e 3,9:1, para o sódio, sendo este o único cátion solúvel que diminuiu com a subsolagem.
\end{abstract}

Termos de indexação: salinidade, sodicidade, gesso, esterco de curral, palha de carnaúba, relação catiônica.

\footnotetext{
(1) Parte da Tese de Doutorado do primeiro autor, apresentada à Escola Superior de Agricultura Luiz de Queiroz - ESALQ/USP. Trabalho com suporte financeiro da EMBRAPA. Recebido para publicação em agosto de 1996 e aprovado em maio de 1998.

(2) Pesquisador do sistema EMPARN/EMBRAPA. Caixa Postal 188, CEP 59020-390 Natal (RN).

(3) Professor Titular do Departamento de Ciência do Solo, ESALQ/USP. Caixa Postal 09, CE P 13418-900 Piracicaba (SP).

(4) Professor do Centro de Ciências Agrárias, Universidade Federal do Piauí - UFPI. Campus da Socopos, CEP 64049-550 Teresina (PI ).
} 


\title{
SUMMARY: CHEMICAL PROPERTY CHANGES IN SALINE-SODIC SOIL BY SUBSOILING AND AMENDMENTS
}

\begin{abstract}
Salt-affected soils are characteristics of arid and semi-arid zones of the world. This study was undertaken at Açu val ley regi on of Rio Grande do NorteState, Brazil, by means of a field experiment conducted from April/ 90 to J anuary/91. The objective of this study was to evaluate the effects of subsoiling and amendment additions (gypsum, farmyard manureand Copernica cerifera straw), on chemical properties of salinesodic soil. Subsoiling treatment provided beneficial effects decreasing soil exchangeable sodium percentage(ESP) and $\mathrm{pH}$ leves. Gypsum treatments increased soil exchangeable $\mathrm{Ca}$ and salinity levels and decreased ESP to levels lower than $15 \%$ in soil layer depth to 0-0.15 m. Farmyard manure treatment decreased ESP levels, especially when associated with subsoiling and gypsum treatment. There is a natural imbalance in $\mathrm{K}: \mathrm{Ca}: \mathrm{Mg}$ ratios that increases with gypsum treatments requiring soil potassi um correction in order to reach the equilibrium. Theratios between exchangeableand sol ubl ecations ranged from 13 to 16:1 for cal ci um and magnesium and from 0.3 and 3.9:1 for sodium. Among the soluble cations, sodium was the only one decreased by subsoiling.
\end{abstract}

Index terms: salinity, sodicity, gypsum, farmyard manure, Copernica cerifera straw, cation ratio.

\section{INTRODUÇÃO}

Os solos afetados por sais não têm a mesma dimensão geográfica dos solos ácidos, mas representam $10 \%$ das terras do globo (Szabolcs, 1989). E m termos comparativos, esses sol os situamseem classes opostas, pois, enquanto os sol os ácidos têm $\mathrm{pH}<7$, são deficientes em bases trocáveis, apresentam cátion domi nantefloculante (Al e, ou, H), os problemas são de ordem química e de fácil correção na camada superficial, os sol os afetados por sais geralmente têm $\mathrm{pH}>7$, bases trocáveis em excesso, apresentam cátion dominante dispersante (Na) eos problemas são de natureza química efísica, sendo de difícil manejo e correção. Assim, como a falta de bases trocáveis no solo causa deficiência de nutrientes para as plantas, o excesso de bases pode acarretar desequilíbrios nutricionais ou causar toxidez, constituindo também um sério problema.

A salinidade do sol o émedida pela condutividade elétrica no extrato de saturação ( $C . E_{\text {es }}$ ), enquanto a sodicidade é avaliada pela porcentagem de sódio trocável (PST) no complexo de troca de cátions do solo. Valores de C.E $\mathrm{E}_{\text {es }}$ acima de $4 \mathrm{dS} \mathrm{m}^{-1}$ e de PST acima de 15\% caracterizam o solo como sal ino-sódico (Estados Unidos, 1990).

A correção de solos salino-sódicos compreende o uso de condicionadores químicos e, ou, orgânicos, práticas mecânicas e lavagem dos sais. O gesso é o condicionador químico mais empregado como fonte de cálcio para substituição do sódio trocável, que pode ser lixiviado, com conseqüente redução da sodicidade do solo (Ferreyrah \& Coelho, 1986;
Armstrong \& Tanton, 1992; Santos, 1995), aumentando, dessa forma, o cál cio trocável no solo (Silva, 1978; Caval cante, 1984; Lucena, 1986; Santos, 1995). O gesso interfere na salinidade do solo, aumentando-a, inicialmente, pela liberação de el etrólitos e diminuindo-a, no decorrer do tempo, por melhorias na permeabilidade que favorecem a lixiviação de sais (Gupta \& Bajpai, 1977; Gheyi et al., 1995 e Santos, 1995). Nos solos salino-sódicos, por serem alcalinos, a disponibilidade relativa de nutrientes pode ser afetada (Oliveira, 1988); Malavolta et al., 1993); no entanto, a condição pode ser amenizada pelo uso do gesso (Singh \& Singh, 1989; Dubey \& Mondal, 1994; Santos, 1995).

Práticas mecânicas, como subsol agem, aumentam a infiltração de água no solo e favorecem a redução da salinidade e sodicidade do solo (Silva, 1978); quando associadas à gessagem, promovem a adequação dessas características em toda a zona radicular (Rasmussen et al., 1972).

Os condicionadores orgânicos (como fonte de cál cio emagnésio) reduzema PST do solo, sendo mais eficiente o esterco de curral (Puttaswamygowda \& Pratt, 1973). Podem, no entanto, apresentar al guma inconveniência; como fonte biológica de sais, em quantidades el evadas podem contribuir para a ocorrência de desequilíbrios nutricionais (Ayers \& Westcot, 1991; Kovda \& Minashina, citados por Szabolcs, 1989).

Este trabal ho teve como objetivo avaliar o efeito de condicionadores e da subsolagem em algumas características químicas de um solo aluvial salinosódico. 


\section{MATERIAL E MÉTODOS}

A pesquisa foi realizada em solo aluvial salinosódico de textura franco-siltosa, da região do baixo Açu (RN) e consistiu de dois sistemas de manejo do solo (SS = preparo convencional com aração + gradagem eCS = preparo convencional +subsolagem a $0,50 \mathrm{~m}$ de profundidade) e da aplicação de condicionadores: $(G)=$ gesso -30 t ha-1 $^{-1}(E)=$ esterco de curral (20 t ha-1, base seca), (G +E) = gesso (30 t ha-1) +esterco (20 t ha-1), (P) =palha decarnaúba (20 t ha-1, base seca), $(P+G)=$ palha de carnaúba (20 t ha-1) +gesso (30 t ha-1) e (T) =testemunha (sem condicionador).

O efeito do preparo do sol o foi avaliado como experimentos em grupo, com dois ensaios instalados lado a lado, separados por uma faixa de $5 \mathrm{~m}$ sem cultura. O delineamento dos ensaios foi em blocos ao acaso, em esquema de parcel as subdi vididas, com seis tratamentos (condicionadores), duas profundidades do sol o- subparcelas (0-0,15 e 0,15-0,30 m), com quatro repetições. As parcelas foram dimensionadas de 6,0 x 10,0 m, com $36 \mathrm{~m}^{2}$ de área útil, perfazendo cada ensaio $2.700 \mathrm{~m}^{2}$, incluindo faixas em branco de $5 \mathrm{~m}$ entre os blocos e $3 \mathrm{~m}$ entre as parcelas, para prevenir riscos de interferências entre os tratamentos testados.

A subsolagem foi realizada antes da aplicação dos condicionadores, a uma profundidade de $0,50 \mathrm{~m}$ e com espaçamento de $1 \mathrm{~m}$, utilizando trator de pneu com subsolador de duas hastes tipo bico-de-pato.

Os condicionadores foram aplicados a lanço e incorporados com grade aradora, em abril/1990. A dosagem de gesso empregada correspondeu à recomendação integral para redução da PST a 15\% na camada de 0-0,40 m, estimada com base na PST atual e na CTC do solo, conforme método descrito em Pizarro (1985).

Os ensaios foram realizados sob condições naturais de drenagem e compreenderam amostragens de sol o com trado de "caneco", fazendosecinco amostras simples por parcela, para formação de uma amostra composta. As amostras de sol o foram col etadas em camadas de 0-0,15 e 0,15-0,30 m, aos nove meses da aplicação dos tratamentos, determinando-se $\mathrm{pH}$, condutividade elétrica do extrato saturado do solo ( $\left(\mathrm{C}_{\mathrm{es}}\right)$, cátions extraíveis, solúveis e trocáveis e cálculo da PST.

$\mathrm{O} \mathrm{pH}$ foi determinado em água $(1: 2,5)$, e os cátions, extraíveis em solução de acetato de amônio $1 \mathrm{~mol} \mathrm{~L}^{-1}$ (pH 7,0). O preparo, a extração e as determinações de cátions solúveis no extrato saturado do solo foram efetuados, utilizando o método preconizado por Richards (1974). As determinações de sódio e potássio foram feitas em fotômetro de chama; as de cálcio e magnésio, em espectrofotômetro de absorção atômica. O hidrogênio foi extraído em acetato de cálcio 0,5 mol L-1 $(\mathrm{pH} \mathrm{7,0)}$ e determinado por titulação com hidróxi do de sódio $0,1 \mathrm{~mol} \mathrm{~L}^{-1}$, usando fenolftal eína como indicador. Os valores de cátions trocáveis foram obtidos por diferença entre extraíveis e solúveis. A porcentagem de saturação desódi otrocável nosolo (PST) foi obtida por mei o da relação da concentração desódio trocável com o total das concentrações de cátions trocáveis, pel o método usual de cál culo: PST $=100$ Na CTC-1.

No período, foi efetuado um cultivo de arroz irrigado por aspersão, com lâmina total de água de cerca de $700 \mathrm{~mm}$. A água usada na irrigação foi classificada como de baixa salinidade, média sodicidade e sem problemas de toxicidade de sódio e, ou, cloro (Pizarro, 1985; Ayers \& Westcot, 1991).

Utilizaram-se a C.E $E_{\text {es }}$ e a PST como medidas de avaliação das condições quími cas do solo, representando os níveis de salinidade e sodicidade; foram adotados, como limites de dasses de comparação, os valores $4 \mathrm{dS} \mathrm{m}^{-1}$ e $15 \%$, respectivamente. Os resultados de cátions trocáveis, pH, C.E $\mathrm{E}_{\mathrm{es}}$ e PST, foram submetidos à análise de variância com teste $F(P<0,05)$ e à comparação de médias pelo teste de Tukey $(P<0,05)$.

\section{RESULTADOS E DISCUSSÃO}

Entre os cátions trocáveis no solo estudado, o cálcio é o mais abundante no complexo de troca, seguido pelo sódio, em condições naturais, sem uso de condicionadores (Quadros 1 e 2). Os resultados de cál ciotrocável foram influenciados significativamente pel o uso de condicionadores e pela profundidade do solo, cuja interação entre ambos foi significativa $(P<0,05)$; os de magnésio tiveram influência apenas da subsolagem; os de sódio, da subsolagem e profundidade, e os de potássio, somente da profundidade, com val ores mais el evados na camada superficial $(P<0,01)$.

O gesso, sozinho ou combinado aos condicionadores orgâni cos, proporcionou os maiores val ores de cál cio trocável, determinados na camada de 0-0,15 m do sol o. Nos demais tratamentos, independentemente da profundidade do solo, e nos tratamentos com gesso, na profundidade de $0,15-0,30 \mathrm{~m}$, os valores de cál cio trocável não diferiram entresi (Quadro 1). Por ser o gesso fonte de cál cio é natural que, com a aplicação desse condicionador, os teores do el emento no solo aumentem na camada de solo incorporada, concordando, dessa forma com os resultados obtidos por Silva (1978), Cavalcante (1984), Lucena (1986) e Santos (1995). A área com subsolagem contém, naturalmente, mais magnésio trocável do que a não subsolada, o que explica os valores mais elevados ali determinados.

Avaliando os teores de potássio, cál cio emagnésio trocáveis no solo, observa-se que, enquanto a relação K:Ca:M g situou-se entre 1:21:7 a 1:35:14, nas áreas 
Quadro 1. Cátions trocáveis em camadas do solo, sob efeito de subsolagem/condicionadores

\begin{tabular}{|c|c|c|c|c|c|c|c|}
\hline \multirow{3}{*}{ Profundidade } & \multirow{3}{*}{ Condicionador $^{(1)}$} & \multicolumn{6}{|c|}{ Cátions trocáveis } \\
\hline & & \multicolumn{2}{|c|}{ Cálcio } & \multicolumn{2}{|c|}{ Magnésio } & \multicolumn{2}{|c|}{ Potássio } \\
\hline & & $\mathbf{S S}^{(2)}$ & $\mathrm{CS}^{(3)}$ & SS $^{(2)}$ & $\mathbf{C S}^{(3)}$ & $\mathbf{S S}^{(2)}$ & $\mathbf{C S}^{(3)}$ \\
\hline $\mathrm{m}$ & & \multicolumn{6}{|c|}{$\longrightarrow \mathrm{mmol}_{\mathrm{c}} \mathrm{kg}^{-1}$} \\
\hline \multirow[t]{6}{*}{$0-0,15$} & G & \multicolumn{2}{|c|}{$152,9 a b$} & $32,7 \mathrm{a}$ & $36,0 \mathrm{a}$ & \multicolumn{2}{|c|}{3,3 a } \\
\hline & $\mathrm{E}$ & \multicolumn{2}{|c|}{$103,9 \mathrm{c}$} & $31,7 \mathrm{a}$ & 42,7 a & \multicolumn{2}{|c|}{$3,8 \mathrm{a}$} \\
\hline & $\mathrm{G}+\mathrm{E}$ & \multicolumn{2}{|c|}{181,3 a } & 34,6 a & $37,2 \mathrm{a}$ & \multicolumn{2}{|c|}{$3,7 \mathrm{a}$} \\
\hline & $P$ & \multicolumn{2}{|c|}{$100,1 \mathrm{c}$} & 35,6 a & $41,0 \mathrm{a}$ & \multicolumn{2}{|c|}{$4,1 \mathrm{a}$} \\
\hline & $G+P$ & \multicolumn{2}{|c|}{$150,5 b$} & $35,8 \mathrm{a}$ & $35,9 \mathrm{a}$ & \multicolumn{2}{|c|}{$3,9 a$} \\
\hline & $\mathrm{T}$ & \multicolumn{2}{|c|}{$93,3 \mathrm{c}$} & $29,1 \mathrm{a}$ & 39,8 a & \multicolumn{2}{|c|}{$3,8 \mathrm{a}$} \\
\hline Média & & \multicolumn{2}{|c|}{$130,3 \mathrm{~A}$} & $33,3 \mathrm{~A}$ & $38,8 \mathrm{~A}$ & \multicolumn{2}{|c|}{$3,8 \mathrm{~A}$} \\
\hline \multirow[t]{6}{*}{$0,15-0,30$} & G & \multicolumn{2}{|c|}{$112,4 \mathrm{c}$} & $32,7 \mathrm{a}$ & $33,1 \mathrm{a}$ & \multicolumn{2}{|c|}{$3,0 \mathrm{a}$} \\
\hline & E & \multicolumn{2}{|c|}{$97,7 \mathrm{c}$} & $26,8 \mathrm{a}$ & $41,4 a$ & \multicolumn{2}{|c|}{$3,4 \mathrm{a}$} \\
\hline & $\mathrm{G}+\mathrm{E}$ & \multicolumn{2}{|c|}{$112,3 \mathrm{c}$} & $32,4 \mathrm{a}$ & $39,4 a$ & \multicolumn{2}{|c|}{$3,1 \mathrm{a}$} \\
\hline & $P$ & \multicolumn{2}{|c|}{$94,9 \mathrm{c}$} & 31,5 a & $38,7 \mathrm{a}$ & \multicolumn{2}{|c|}{$3,0 \mathrm{a}$} \\
\hline & $G+P$ & \multicolumn{2}{|c|}{$107,0 \mathrm{c}$} & $32,4 a$ & 37,8 a & \multicolumn{2}{|c|}{$3,0 \mathrm{a}$} \\
\hline & $\mathrm{T}$ & & & $30,1 \mathrm{a}$ & 36,3 a & & \\
\hline Média & & & & $31,0 \mathrm{~A}$ & $37,8 \mathrm{~A}$ & & \\
\hline Média de subsolagem & & $114,5 \mathrm{~A}$ & $118,8 \mathrm{~A}$ & $32,1 \mathrm{~A}$ & $38,3 \mathrm{~B}$ & $3,6 \mathrm{~A}$ & $3,3 \mathrm{~A}$ \\
\hline Valor $F^{(4)}$ Subs. (S) & & & $7^{\text {ns }}$ & & & & \\
\hline Valor F Cond. (C) & & & $5 * *$ & & & & \\
\hline Valor F Prof. (P) & & & $9 * *$ & & & & \\
\hline Valor $\mathrm{F}(\mathrm{C} \times \mathrm{P})$ & & & $0 * *$ & & & & \\
\hline C.V. Cond. (\%) & & & & 23 & & 28 & \\
\hline C.V. Prof. (\%) & & & & 16 & & 20 & \\
\hline
\end{tabular}

${ }^{(1)} \mathrm{G}$ :Gesso; E:Esterco de curral; P:Palha de carnaúba; T:Testemunha. ${ }^{(2)} \mathrm{SS}$ : sem subsolagem; ${ }^{(3)} \mathrm{CS}$ : com subsolagem. ${ }^{(4)} \mathrm{Consideradas}$ apenas as interações significativas. Médias de tratamentos ou interações: ${ }^{\text {ns }}$ não diferem entre si $(P<0,05)$; ${ }^{*}$ diferem $(P<0,05)$; ** diferem $(P<0,01)$. Para determinado el emento e comparações no sentido vertical, médias seguidas de mesma letra minúscula, em uma mesma profundidade do solo, e de letras maiúsculas, para as duas profundidades, não diferem entre si $(P<0,05)$.

testemunha e só com a incorporação de condicionadores orgânicos; nos tratamentos com gesso e combinações, tal relação variou de 1:33:10 a 1:55:11 (Quadro 2). Naturalmente existe um desbalanço que se acentua com a gessagem, necessitando de uma correção do potássio trocável do solo para se estabelecer um equilíbrio em proporções mais adequadas para a maioria das culturas, situando a relação entre 1:9:3 e 1:25:5 (Potash \& Phosphatel Institute, citado por Malavolta et al., 1993). O desequilíbrio nas rel ações de cátions, nessas áreas, é admitido por Oliveira (1988) como o problema mais importante para o estabelecimento de culturas, superando a própria saturação com sódio.

A subsolagem sozinha contribuiu para uma redução superior a 48\%, em valores relativos, na PST da camada de 0-0,15 m do solo, em relação à testemunha (Quadro 3). O efeito da subsolagem na diminuição do sódi otrocável ea conseqüente redução da PST reforçam a hipótese de existência de camadas impermeáveis no solo, condição mais limitante para redução da sodicidade. A subsolagem, rompendo parte dessas camadas, favorecea infiltração da água, com o deslocamento de sais para camadas mais profundas do solo, no que concordam os resultados de Rasmussen et al. (1972) e Silva (1978). Como a PST é maior na camada mais profunda do solo $(P<0,01)$, é provável que a condição de umi dade e o tempo decorrido da aplicação dos tratamentos não tenham favorecido o deslocamento de cátions para profundidades maiores que 0,15-0,30 m.

No rol dos cátions trocáveis, pela sua valência e raio iônico hidratado, o sódio situa-senoúltimolugar 
Quadro 2. Valores de pH e relação entre cátions trocáveis nas duas camadas do solo, sob efeito de subsolagem/condicionadores

\begin{tabular}{|c|c|c|c|c|c|}
\hline \multirow{2}{*}{ Profundidade } & \multirow{2}{*}{ Condicionador(1) } & \multicolumn{2}{|c|}{ pH } & \multicolumn{2}{|c|}{ Relação K:Ca:Mg } \\
\hline & & $\mathbf{S S}^{(2)}$ & CS $^{(3)}$ & $\mathbf{S S}^{(2)}$ & $\mathbf{C S}^{(3)}$ \\
\hline \multicolumn{6}{|l|}{$\mathrm{m}$} \\
\hline \multirow[t]{6}{*}{$0-0,15$} & G & 7,4 a & $6,8 \mathrm{a}$ & $1: 50: 10$ & 1:43:11 \\
\hline & E & $8,1 \mathrm{a}$ & $6,8 \mathrm{a}$ & 1:29:09 & $1: 26: 10$ \\
\hline & $G+E$ & $7,0 \mathrm{a}$ & $6,7 \mathrm{a}$ & 1:44:09 & $1: 55: 11$ \\
\hline & $\mathrm{P}$ & $7,4 \mathrm{a}$ & $6,8 \mathrm{a}$ & $1: 22: 08$ & 1:29:12 \\
\hline & $G+P$ & 6,9 a & $6,7 a$ & $1: 40: 10$ & $1: 37: 09$ \\
\hline & $\mathrm{T}$ & $8,0 \mathrm{a}$ & 6,8 a & $1: 21: 07$ & $1: 29: 11$ \\
\hline Média & & $7,5 \mathrm{~A}$ & $6,8 \mathrm{~A}$ & 1:34:09 & $1: 37: 11$ \\
\hline \multirow[t]{6}{*}{$0,15-0,30$} & G & $7,9 \mathrm{a}$ & $7,5 \mathrm{a}$ & 1:39:10 & $1: 36: 12$ \\
\hline & E & $8,2 \mathrm{a}$ & $6,7 \mathrm{a}$ & 1:29:09 & $1: 29: 12$ \\
\hline & $G+E$ & 7,7 a & 6,9 a & $1: 33: 10$ & $1: 40: 13$ \\
\hline & $\mathrm{P}$ & $7,8 \mathrm{a}$ & 6,9 a & $1: 29: 10$ & $1: 35: 14$ \\
\hline & $G+P$ & $7,5 \mathrm{a}$ & $7,0 \mathrm{a}$ & 1:35:11 & $1: 38: 13$ \\
\hline & $\mathrm{T}$ & 7,9 a & $7,2 \mathrm{a}$ & $1: 26: 08$ & $1: 34: 13$ \\
\hline Média & & $7,8 \mathrm{~B}$ & $7,0 \mathrm{~B}$ & $1: 32: 10$ & $1: 35: 13$ \\
\hline Média de subsolagem & & $7,6 \mathrm{~A}$ & $6,9 \mathrm{~B}$ & 1:32:09 & $1: 36: 12$ \\
\hline Valor $\mathrm{F}^{(4)}$ Subs. (S) & & \multicolumn{2}{|c|}{$22,66 * *$} & & \\
\hline Valor F Cond. (C) & & \multicolumn{2}{|c|}{$0,98^{\text {ns }}$} & & \\
\hline Valor F Prof. (P) & & $15,84^{* *}$ & $15,94^{* *}$ & & \\
\hline Valor $\mathrm{F}(\mathrm{C} \times \mathrm{P})$ & & & & & \\
\hline C.V. Cond. (\%) & & & & & \\
\hline C.V. Prof. (\%) & & & & & \\
\hline
\end{tabular}

(1)G:Gesso; E:Esterco de curral; P:Palha de carnaúba; T:Testemunha. ${ }^{(2)}$ SS: sem subsolagem; ${ }^{(3)} \mathrm{CS}$ : com subsolagem. ${ }^{(4)} \mathrm{Consideradas}$ apenas as interações significativas. Médias de tratamentos ou interações: ${ }^{n s}$ não diferem entre si $(P<0,05)$; ${ }^{*}$ diferem $(P<0,05)$; **diferem $(\mathrm{P}<0,01)$. Médias de $\mathrm{pH}$ seguidas de mesma letra no sentido vertical, em cada profundidade do solo, e no sentido horizontal, para a variável subsolagem, não diferem entre si $(P<0,05)$

quanto à seletividade de adsorção (série liotrópica), o que em si é um fator favorável à sua substituição, pois, em condições de igual dade de concentração, é adsorvido na sobra de cargas após o preenchimento por $\mathrm{H}^{+}>>\mathrm{Al}^{3+}>\mathrm{Ca}^{2+}>\mathrm{Mg} \mathrm{g}^{2+}>\mathrm{K}^{+}>\mathrm{NH}_{4}{ }^{+}>\mathrm{Na}^{+} . \mathrm{O}$ hidrogênioé uma exceção, tem menor massa atômica, mas alta energia de adsorção, da ordem de quatro vezes a do cál cio e 17 vezes a do sódio (Yagodin, 1984). Assim, a oportunidade para o sódio se deslocar no perfil do solo é incrementada pela subsolagem.

Em todos os tratamentos com gesso, a PST na camada de $0-0,15 \mathrm{~m}$ do solo ficou abaixo de $15 \%$ (Quadro 3). Na área sem subsolagem, o efeito dos condicionadores na PST ficou restrito à camada de $0-0,15 \mathrm{~m}$, proporcionando à combinação do gesso + esterco decurral menor val or dePST do que o obtido na testemunha ou na área só com o esterco de curral $(P<0,05)$. Com gesso sozinho, a PST foi inferior à determinada na testemunha $(P<0,05)$, concordando com as reduções de PST obtidas por Ferreyrah \& Coelho (1986), Armstrong \& Tanton (1992) e Santos (1995), sendo explicadas pelo aumento do cálcio trocável, acentuando as reações de troca com substituição do sódio trocável no compl exo sortivo do sol o. Na área subsolada, existem fortes indícios de melhorias na PST pelo uso combinado de gesso + orgânicos, nas duas camadas de solo. Na camada de 0,15-0,30 m do solo, o efeito do esterco de curral favoreceu para menor valor de PST do que nos tratamentos testemunha e só com palha de carnaúba $(P<0,05)$.

Os condicionadores orgânicos foram mais benéficos, quando associados ao gesso. Conforme afirmam Puttaswamygowda \& Pratt (1973), o esterco de curral foi o mais eficiente dos condicionadores orgânicos, especialmente na área subsolada, 
provavel mente por ser de mais fácil decomposição e o seu efeito salinizante ser de curta duração. Não se constatou inconveniência do uso dos condicionadores orgânicos, como al ertaram Ayers \& Westcot (1991) e Kovda \& Minashina, citados por Szabol cs (1989).

$\mathrm{O}$ pH da área subsolada foi menor do que o da não subsolada ( $P<0,01)$, aparentementesem relação com os condicionadores aplicados (Quadro 2). Sem subsolagem, os valores de $\mathrm{pH}$ na profundidade de 0-0,15 m tendem a ser menores nas áreas tratadas com gesso, indicando que o mesmo contribui para o abaixamento do $\mathrm{pH}$. Reduzindo a alcal inidade, quer por gessagem quer por subsolagem, previnem-se os problemas de disponibilidade relativa de nutrientes no solo relatados por Oliveira (1988) e Malavolta et al. (1993). As diminuições no pH do solo, em valores absolutos, concordam com os resultados obtidos por
Singh \& Singh (1989), Dubey \& Mondal, (1994) e Santos (1995) e se justificam pelo deslocamento de bases trocáveis (sódio) para camadas mais profundas, possibilitando a ocupação de parte dos sítios de troca por hidrogênio proveniente da dissociação iônica da água; carbonatos e parte dos bicarbonatos, responsáveis pela alcalinidade, formam compl exos de baixa solubili dade com o cálcio, e o próprio aumento da concentração de el etrólitos na solução do solo pode contribuir para o abaixamento do $\mathrm{pH}$.

A gessagem contribuiu para o aumento da sal inidadedo solo na camada de0-0,15 m (Quadro 3). Os valores de $\mathrm{C}$. $\mathrm{E}_{\mathrm{es}}$ nas áreas apenas com condicionadores orgânicos e na testemunha foram menores do que na com gesso sozinho ou combinado com esterco de curral $(P<0,05)$; mesmo assim, o solo

Quadro 3. Parâmetros químicos relacionados com a afetação de sais no solo sob efeito do manejo com e sem subsolagem e do uso de condicionadores

\begin{tabular}{|c|c|c|c|c|c|c|c|}
\hline \multirow[b]{2}{*}{ Profundidade } & \multirow{2}{*}{ Condicionador $^{(1)}$} & \multicolumn{2}{|c|}{ C.E $\mathbf{e s}_{\mathrm{es}}$} & \multicolumn{2}{|c|}{ Sódio } & \multicolumn{2}{|c|}{ PST } \\
\hline & & $\mathbf{S S}^{(2)}$ & $\mathrm{CS}^{(3)}$ & $\mathbf{S S}^{(2)}$ & $\mathbf{C S}^{(3)}$ & SS $^{(2)}$ & $\mathbf{C S}^{(3)}$ \\
\hline $\mathrm{m}$ & & \multicolumn{2}{|c|}{$\mathrm{dS} \mathrm{m}^{-1}$} & \multicolumn{2}{|c|}{$-\mathrm{mmol}_{\mathrm{c}} \mathrm{kg}^{-1}$} & \multicolumn{2}{|c|}{$\%$} \\
\hline \multirow[t]{6}{*}{$0-0,15$} & G & \multicolumn{2}{|c|}{$6,44 a$} & 35,5 a & $20,8 \mathrm{a}$ & $14,33 \mathrm{bc}$ & $9,70 \mathrm{a}$ \\
\hline & $E$ & \multicolumn{2}{|c|}{$3,72 \mathrm{~b}$} & 71,7 a & 26,9 a & $31,72 a b$ & $13,81 \mathrm{a}$ \\
\hline & $G+E$ & \multicolumn{2}{|c|}{$6,31 \mathrm{a}$} & 24,6 a & 18,4 a & $10,38 \mathrm{c}$ & $7,46 \mathrm{a}$ \\
\hline & $\mathrm{P}$ & \multicolumn{2}{|c|}{$3,68 \mathrm{~b}$} & 56,5 a & 42,7 a & 26,89 abc & $22,17 \mathrm{a}$ \\
\hline & $G+P$ & \multicolumn{2}{|c|}{$5,44 a b$} & 30,0 a & 27,7 a & $12,90 \mathrm{bc}$ & $12,73 \mathrm{a}$ \\
\hline & $\mathrm{T}$ & \multicolumn{2}{|c|}{$4,07 \mathrm{~b}$} & $73,1 \mathrm{a}$ & 36,7 a & 35,99 a & $18,64 \mathrm{a}$ \\
\hline Média & & \multicolumn{2}{|c|}{$4,94 \mathrm{~A}$} & $48,2 \mathrm{~A}$ & $28,9 \mathrm{~A}$ & $22,04 \mathrm{~A}$ & $14,09 \mathrm{~A}$ \\
\hline \multirow[t]{6}{*}{$0,15-0,30$} & G & \multicolumn{2}{|c|}{$4,89 \mathrm{a}$} & 70,2 a & 69,5 a & 28,96 a & $32,43 a b$ \\
\hline & $E$ & \multicolumn{2}{|c|}{$3,14 a$} & 92,9 a & 35,2 a & 40,93 a & $17,21 \mathrm{~b}$ \\
\hline & $G+E$ & \multicolumn{2}{|c|}{$4,44 a$} & 83,9 a & 43,6 a & 36,37 a & $19,29 a b$ \\
\hline & $\mathrm{P}$ & \multicolumn{2}{|c|}{$3,72 \mathrm{a}$} & 91,3 a & 89,8 a & 39,69 a & 36,76 a \\
\hline & $G+P$ & \multicolumn{2}{|c|}{$4,21 \mathrm{a}$} & 71,6 a & 62,0 a & $31,61 \mathrm{a}$ & $26,79 a b$ \\
\hline & $\mathrm{T}$ & \multicolumn{2}{|c|}{$4,08 \mathrm{a}$} & 85,0 a & 87,7 a & 37,45 a & $37,29 \mathrm{a}$ \\
\hline Média & & \multicolumn{2}{|c|}{$4,08 \mathrm{~B}$} & $82,5 \mathrm{~B}$ & $64,6 \mathrm{~B}$ & $35,84 \mathrm{~B}$ & $28,30 \mathrm{~B}$ \\
\hline \multicolumn{2}{|l|}{ Média de subsolagem } & $4,76 \mathrm{~A}$ & $4,39 \mathrm{~A}$ & $65,3 \mathrm{~A}$ & $45,9 \mathrm{~B}$ & $29,57 \mathrm{~A}$ & $21,29 \mathrm{~B}$ \\
\hline \multicolumn{2}{|l|}{ Valor $\mathrm{F}^{(4)}$ Subs. (S) } & \multicolumn{2}{|c|}{$1,85^{\text {ns }}$} & \multicolumn{2}{|c|}{$7,14^{*}$} & \multicolumn{2}{|c|}{$7,03 *$} \\
\hline Valor F Cond. (C) & & \multicolumn{2}{|c|}{$7,91^{*}$} & \multicolumn{2}{|c|}{$1,95^{\mathrm{ns}}$} & $2,96 *$ & $2,73^{*}$ \\
\hline Valor F Prof. (P) & & \multicolumn{2}{|c|}{$14,34 * *$} & \multicolumn{2}{|c|}{$41,6 * *$} & 40,6 & $5 * *$ \\
\hline Valor $F(C \times P)$ & & & & & & 0,7 & \\
\hline C.V. Cond. (\%) & & 25 & & & & 32,5 & \\
\hline C.V. Prof. (\%) & & & & & & 22,7 & \\
\hline
\end{tabular}

${ }^{(1)} \mathrm{G}$ :Gesso; E:Esterco de curral; P:Palha de carnaúba; T:Testemunha. ${ }^{(2)}$ SS: sem subsolagem; ${ }^{(3)} \mathrm{CS}$ : com subsolagem. ${ }^{(4)}$ Consideradas apenas as interações significativas. Médias de tratamentos ou interações: ${ }^{\text {ns }}$ não diferem entre si $(P<0,05) ; *$ diferem $(P<0,05)$; $* *$ diferem $(P<0,01)$. Para determinado elemento, médias seguidas de mesma letra maiúscula na horizontal não diferem entre si $(P<0,05)$. Para uma mesma profundidade do solo, médias de $C . E_{\text {es }}$ (condutividade elétrica no extrato de saturação) e numa mesma coluna médias de sódio ou PST (porcentagem de sódio trocável) seguidas de mesma letra, não diferem entre si $(P<0,05)$. 
continua salino ou próximo do limite de salinidade (C. $E_{\mathrm{es}} \geq 4 \mathrm{dS} \mathrm{m}^{-1}$ ) em todas as situações, o que poderá ser contornado com um aumento na lâmina de água de irrigação. O gesso, como fonte de el etrólitos para a solução do solo, é natural que, inicialmente, aumentea sua salinidade. Nesse sentido, concordam os trabalhos de Gupta \& Bajpai (1977) e Santos (1995); entretanto, ao longo do tempo, exercendo efeito amenizador na impermeabilização do sol o pela substituição do cátion dispersante $(\mathrm{Na})$ por um floculante (Ca), aumentará a sua condutividade hidráulica, favorecendo a infiltração da água, com diminuição da salinidade do solo, conforme observaram Gheyi et al. (1995).

Quanto aos cátions solúveis, o sódio lidera, assumindo em torno de 70\% do total (Quadro 4). A subsolagem diminuiu o sódio solúvel no solo e favoreceu para que sua concentração média não diferisse entre as duas profundidades $(P<0,05)$. $O$ cálcio e o potássio solúveis apresentaram maiores concentrações na camada de $0-0,15 \mathrm{~m}$ do solo e, juntamente com o sódio, tiveram os valores mais el evados sempre associados à prática da gessagem (Quadro 4). As proporções entre cátions trocáveis e solúveis evidenciam o maior poder tampão do cálcio e magnésio, situando-se entre 13 e 16:1, na camada de $0-0,15 \mathrm{~m}$ do solo. Para o sódio, tais proporções variaram de 0,3 a 3,9:1 e foram influenciadas pela presença do gesso que, como fonte de cál cio, acentua as reações de troca, reduz o sódio trocável e aumenta o sódio solúvel.

\section{CONCLUSÕES}

1. A gessagem, sozinha ou combinada ao uso de condicionadores orgânicos, proporcionou maiores val ores de cálcio trocável no solo, reduziu a PST para menos de $15 \%$ na camada de $0-0,15$ m eaumentou a salinidade.

\section{Quadro 4. Composi ção de cátions solúveis em camadas do solo sob efeito do manejo com e sem subsolagem} e do uso de condicionadores

\begin{tabular}{|c|c|c|c|c|c|c|c|c|c|}
\hline \multirow{3}{*}{ Profundidade } & \multirow{3}{*}{ Condicionador $(1)$} & \multicolumn{8}{|c|}{ Cátions solúveis } \\
\hline & & \multicolumn{2}{|c|}{ Cálcio } & \multicolumn{2}{|c|}{ Magnésio } & \multicolumn{2}{|c|}{ Sódio } & \multicolumn{2}{|c|}{ Potássio } \\
\hline & & SS $^{(2)}$ & $\mathrm{CS}^{(3)}$ & $S S^{(2)}$ & $C S^{(3)}$ & SS ${ }^{(2)}$ & $\mathrm{CS}^{(3)}$ & $\mathbf{S S}^{(2)}$ & $\mathrm{CS}^{(3)}$ \\
\hline $\mathrm{m}$ & & & 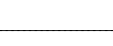 & 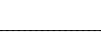 & $\mathrm{mmo}$ & $\mathrm{ckg}^{-1}$ & 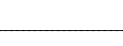 & & \\
\hline \multirow[t]{6}{*}{$0-0,15$} & G & \multicolumn{2}{|c|}{11,3 a } & \multicolumn{2}{|c|}{$2,6 a$} & $39,0 a b$ & $27,6 \mathrm{a}$ & \multicolumn{2}{|c|}{$0,39 a$} \\
\hline & $E$ & \multicolumn{2}{|c|}{$7,1 \mathrm{~b}$} & \multicolumn{2}{|c|}{$2,5 \mathrm{a}$} & $24,1 \mathrm{bc}$ & $15,7 \mathrm{~b}$ & \multicolumn{2}{|c|}{$0,24 a b$} \\
\hline & $G+E$ & \multicolumn{2}{|c|}{$13,1 \mathrm{a}$} & \multicolumn{2}{|c|}{$2,6 a$} & $46,7 \mathrm{a}$ & $22,1 \mathrm{ab}$ & \multicolumn{2}{|c|}{$0,39 a$} \\
\hline & $\mathrm{P}$ & \multicolumn{2}{|c|}{$7,2 \mathrm{~b}$} & \multicolumn{2}{|c|}{$2,7 \mathrm{a}$} & $23,7 \mathrm{c}$ & $18,7 a b$ & \multicolumn{2}{|c|}{$0,19 \mathrm{~b}$} \\
\hline & $G+P$ & \multicolumn{2}{|c|}{$10,8 \mathrm{a}$} & \multicolumn{2}{|c|}{$2,6 \mathrm{a}$} & 29,4 bc & $17,7 \mathrm{~b}$ & \multicolumn{2}{|c|}{$0,36 a b$} \\
\hline & $\mathrm{T}$ & \multicolumn{2}{|c|}{$6,1 \mathrm{~b}$} & \multicolumn{2}{|c|}{$2,3 a$} & $18,8 \mathrm{c}$ & $20,3 a b$ & \multicolumn{2}{|c|}{$0,15 \mathrm{~b}$} \\
\hline M édia & & \multicolumn{2}{|c|}{$9,3 \mathrm{~A}$} & \multicolumn{2}{|c|}{$2,6 \mathrm{~A}$} & $30,3 \mathrm{~A}$ & $20,4 \mathrm{~A}$ & \multicolumn{2}{|c|}{$0,29 \mathrm{~A}$} \\
\hline \multirow[t]{6}{*}{$0,15-0,30$} & G & \multicolumn{2}{|c|}{$7,9 \mathrm{~b}$} & \multicolumn{2}{|c|}{$2,3 a$} & 33,4 a & 24,5 a & 0,2 & $0 \mathrm{~b}$ \\
\hline & $E$ & & & & & $18,3 \mathrm{~b}$ & $12,8 \mathrm{~b}$ & 0,1 & $2 \mathrm{~b}$ \\
\hline & $G+E$ & & & & & $24,8 a b$ & $25,1 \mathrm{a}$ & 0,1 & $7 \mathrm{~b}$ \\
\hline & $P$ & & & & & $23,7 \mathrm{ab}$ & $17,5 a b$ & 0,1 & $6 \mathrm{~b}$ \\
\hline & $G+P$ & & & & & $27,3 a b$ & $19,2 a b$ & 0,1 & $8 \mathrm{~b}$ \\
\hline & $\mathrm{T}$ & & & & & $19,4 \mathrm{~b}$ & $21,0 a b$ & 0,1 & $2 \mathrm{~b}$ \\
\hline M édia & & & B & & & $24,5 \mathrm{~B}$ & $20,0 \mathrm{~A}$ & 0,1 & $6 \mathrm{~B}$ \\
\hline M édia de subsolagem & & $8,4 \mathrm{~A}$ & $8,0 \mathrm{~A}$ & $2,4 \mathrm{~A}$ & $2,6 \mathrm{~A}$ & $27,4 \mathrm{~A}$ & $20,2 \mathrm{~B}$ & $0,22 \mathrm{~A}$ & $0,22 \mathrm{~A}$ \\
\hline Valor $\mathrm{F}^{(4)}$ Subs. (S) & & & $5^{\text {ns }}$ & & $2^{\text {ns }}$ & $13,61 *$ & & 0,0 & $08^{\text {ns }}$ \\
\hline Valor F Cond. (C) & & 15, & $9 * *$ & & $8^{\text {ns }}$ & $4,96 * *$ & $4,13^{*}$ & 5,3 & \\
\hline Valor F Prof. (P) & & 46 & $9 * *$ & & $0^{\text {ns }}$ & $8,40 * *$ & $0,16^{\text {ns }}$ & 57,6 & $6 * *$ \\
\hline Valor $\mathrm{F}(\mathrm{C} \times \mathrm{P})$ & & & $0 * *$ & & $1^{\text {ns }}$ & $1,56^{\mathrm{ns}}$ & $1,53^{\text {ns }}$ & 2,4 & \\
\hline C.V. Cond. (\%) & & 21 & & 25 & & 30,15 & 28,64 & 56,9 & \\
\hline C.V. Prof. (\%) & & 17 & & 15 & & 18,70 & 14,14 & 40,8 & \\
\hline
\end{tabular}

${ }^{(1)}$ G:Gesso; E:Esterco de curral; P:Palha de carnaúba; T:Testemunha. ${ }^{(2)}$ SS: sem subsolagem; ${ }^{(3)} \mathrm{CS}$ : com subsolagem. ${ }^{(4)} \mathrm{Consideradas}$ apenas as interações significativas. Médias de tratamentos ou interações: ${ }^{\text {ns }}$ não diferem entre si $(P<0,05) ; *$ diferem $(P<0,05)$; $* *$ diferem $(P<0,01)$. Para determinado el emento enuma mesma col una, médias seguidas de mesma letra não diferem entresi $(P<0,05)$. 
2. O preparo do solo envolvendo subsolagem reduziu a porcentagem de sódio trocável (PST) e abaixou o pH do solo.

3. O esterco de curral foi benéfico na redução da PST, principal mente quando associado à subsolagem eà gessagem.

4. A gessagem acentuou o desbalanço natural das relações de cátions trocáveis $\mathrm{K}: \mathrm{Ca}: \mathrm{Mg}$ do solo, necessitando de correção potássica.

5. Entre os cátions trocáveis, o cálcio é o mais abundante; entre os cátions solúveis, o sódio é o de maior concentração e foi o único que diminuiu com a subsolagem.

6. Na camada de $0-0,15 \mathrm{~m}$ do solo, o cálcio e magnésio trocáveis ficaram numa proporção entre 13 e 16:1, em relação às formas solúveis, enquanto para o sódio essa relação situou-se entre 0,3 e 3,9:1.

\section{LITERATURA CITADA}

ARMSTRONG, A.S.B. \& TANTON, T.W. Gypsum applications to aggregated saline-sodic clay topsoils. J. Soil Sci., 43:249260, 1992.

AYERS, R.S. \& WESTCOT, D.W. A qualidade da água na agricultura. Tradução de GHEYI, H. R., MEDEIROS, J.F. \& DAMASCENO, F.A.V. Campina Grande, UFPB, 1991. 218p. (FAO. Irrigação e Drenagem, 29)

CAVALCANTE, L.F. E feito do fosfogesso em sol os salinizados da Paraíba cultivados com feijão vigna. Piracicaba, Escola Superior de Agricultura "Luiz de Queiroz", Universidade de São Paulo, 1984. 94p. (Tese de Doutorado)

DUBEY, S.K. \& MONDAL, R.C. Effect of amendments and saline irrigation water on soil properties and yields of rice and wheat in a highly sodic soil. J . Agric. Sci., 122:351-57, 1994.

ESTADOS UNIDOS. Department of Agriculture. Keys to soil taxonomy. 4.ed. Washington, USDA, 1990. 422p. (USDA. SMSS Technical Monograph, 6)

FERREYRAH, F.F. \& COELHO, M.A. Efeito de dose de gesso e subsolagem na produtividade de arroz em solo sódico. R. Bras. Ci. Solo, 10:157-161, 1986.
GHEYI, H.R.; AZEVEDO, N.C.; BATISTA, M.A.F. \& SANTOS, J.G.R. Comparação de métodos na recuperação de solo salino-sódico. R. Bras. Ci. Solo, 19:173-178, 1995.

GUPTA, B.R. \& BAJPAI, P.D. Effect of some inorganic ameliorants on reclamation and phosphorus availability in salt-affected soils. Ind. J . Agric. Res., 11:87-103, 1977.

LUCENA, E. R. Efeito da aplicação do fosfogesso em um solo salino-sódico. Piracicaba, Escola Superior de Agricultura "Luiz de Queiroz", Universidade de São Paulo, 1986. 94p. (Tese de Mestrado)

MALAVOLTA, E.; MURAOKA,T. \& BOARETTO,A.E. O solo como um meio para o crescimento das plantas. Piracicaba, CENA/ USP, 1993. 47p.

OLIVEIRA, M. Os solos e o ambiente agrícola no sistema Piranhas-Açu. Mossoró: ESAM/FGD, 1988. 314p. (Coleção Mossoroense, 380)

PIZARRO, F. Drenaje agricola y recuperación de suel os salinos. Madri, Agricola Española, 1985. 542p.

PUTTASWAMYGOWDA, B.S. \& PRATT, P.P. Effects of straw, calcium chlorid, and submergence on a sodic soil. Soil Sci. Soc. Am. J ., 37:208-212, 1973.

RASMUSSEN, W.W.; MOORE, D.P. \& ALBAN, L.A. I mprovement of a Solonetzic (slick spot) soil by deep plowing, sub-soiling, and amendments. Soil Sci. Soc. Am. Proc., 36:137-142, 1972.

RICHARDS, L.A. Diagnostico y rehabilitacion de suelos salinos y sodicos. México, Limusa, 1974. 172p.

SANTOS, R.V. Correção de um solo salino-sódico e absorção de nutrientes pelo feijão macassar (Vigna unguiculata L. Walp.). Piracicaba, Escola Superior de Agricultura "Luiz de Queiroz", Universidade de São Paulo, 1995. 117p. (Tese de Doutorado)

SILVA, M. J. Efeito de métodos de recuperação em solo com problemas de sais no projeto de irrigação de São GonçaloPB. Viçosa, Universidade Federal deViçosa. 1978. 54p. (Tese de Mestrado)

SINGH, M.V.; SINGH, K.N. Reclamation techniques for improvement of sodic soils and crop yield. Ind. J . Agric. Sci., 59:495-500, 1989.

SZABOLCS, I. Salt-affected soils. Washington, Library of Congress, 1989. 274p.

YAGODIN, B. A. Agricultural chemistry. Moscow, Mir Publishers, 1984. 375p. 